\title{
Laboratory Aspects of Donor Screening for Fecal Microbiota Transplantation at a Korean Fecal Microbiota Bank
}

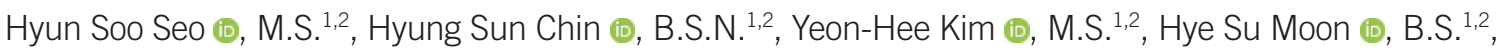

 \\ ${ }^{1}$ Department of Laboratory Medicine and Research Institute of Bacterial Resistance, Yonsei University College of Medicine, Seoul, Korea; ${ }^{2}$ Microbiotix \\ Corporation, Seoul, Korea; ${ }^{3}$ Brain Korea 21 Plus Project for Medical Science, Yonsei University College of Medicine, Seoul, Korea
}

Fecal microbiota transplantation (FMT) is a widely accepted alternative therapy for Clostridioides difficile infection and other gastrointestinal disorders. Thorough donor screening is required as a safety control measure to minimize transmission of infectious agents in FMT. We report the donor screening process and outcomes at a fecal microbiota bank in Korea. From August 2017 to June 2020, the qualification of 62 individuals as FMT donors was evaluated using clinical assessment and laboratory tests. Forty-six (74\%) candidates were excluded after clinical assessment; high body mass index ( $>25$ ) was the most common reason for exclusion, followed by atopy, asthma, and allergy history. Four of the remaining $16(25 \%)$ candidates failed to meet laboratory test criteria, resulting in a $19 \%$ qualification rate. FMT donor re-qualification was conducted monthly as an additional safety control measure, and only three (5\%) candidates were eligible for repeated donation. As high prevalence of multidrug-resistant organisms (55\%) and Helicobacter pylori $(44 \%)$ were detected in qualified donors during the screening, a urea breath test was added to the existing protocol. The present results emphasize the importance of implementing a donor re-qualification system to minimize risk factors not identified during initial donor screening.

Key Words: Korea, Fecal microbiota transplantation, Fecal microbiota bank, Donor screening
Received: July 20, 2020

Revision received: August 31, 2020

Accepted: January 11, 2021

\section{Corresponding author:}

Dongeun Yong, M.D., Ph.D.

Department of Laboratory Medicine and

Research Institute of Bacterial Resistance, Yonsei University College of Medicine,

50-1 Yonsei-ro, Seodaemun-gu, Seoul 03722, Korea

Tel: $+82-2-2228-2442$

Fax: +82-2-364-1583

E-mail: deyong@yuhs.ac

\section{(i) $\$$}

(c) Korean Society for Laboratory Medicine

This is an Open Access article distributed under the terms of the Creative Commons Attribution Non-Commercial License (https://creativecommons.org/licenses/by-nc/4.0) which permits unrestricted non-commercial use, distribution, and reproduction in any medium, provided the original work is properly cited.
Fecal microbiota transplantation (FMT) is widely used as a last resort treatment for Clostridioides difficile infection; it has also been demonstrated to be an effective treatment for other gastrointestinal disorders [1-3]. The increasing demand for FMT has led to the establishment of several fecal microbiota banks around the world to provide safe access to donor feces. Several reports have outlined FMT donor-screening criteria for minimizing the risk of infectious agent transmission [4-10]. A recent report on serious adverse effects associated with extended spectrum $\beta$-lactamase (ESBL)-producing Enterobacteriaceae transmission via FMT emphasizes the need for rigorous FMT donor screening [11]. Implementation of a standardized FMT donor screening protocol is important; however, some modifications to the protocol may be required when considering the local prevalence of infectious agents [8].

Many European and North American fecal microbiota banks have recently reported their donor screening outcomes, and these data have provided insights for establishing evidencebased FMT consensus reports [4-10, 12]. However, there is a paucity of such reports from Asian fecal microbiota banks, despite there being numerous publications on FMT clinical outcomes $[13,14]$. This report presents the donor screening outcomes from a fecal microbiota bank in Korea (Microbiotix Corporation, Seoul, Korea). 
In November 2016, Microbiotix Corporation, a university hospital-affiliated startup company, founded a non-profit fecal microbiota bank in collaboration with physicians from various disciplines (laboratory medicine, gastroenterology, pulmonology, and infectious disease). This fecal microbiota banking project was approved by the Severance Hospital Institutional Review Board, Seoul, Korea (IRB No. 4-2016-0850). Donors were recruited through poster advertisements at the Yonsei University Health System starting August 2017. Individuals interested in fecal donation were invited for a two-stage donor-screening process (stage 1, clinical assessment; stage 2, laboratory tests) created based on the Korean Transfusion Guidelines and the European and American FMT donor screening protocols [4, 5, 9, 15].

A summary of the donor screening criteria is provided in Table 1. All donor candidates provided informed consent to participate in the study. An in-person clinical assessment (stage 1) was carried out to evaluate the donor candidates for general health and gastrointestinal conditions and for any risk factors for transmissible diseases. This process was performed by a nurse clinical research coordinator and was verified by a laboratory medicine specialist. At stage 2, donor candidates underwent serological/fecal screening, a urea breath test (UBT), and chest (posteroanterior) radiography to identify underlying health conditions and potentially transmissible pathogens. Although FMT is recognized as a safe and effective treatment with manageable adverse effects (e.g., bloating, cramping, and diarrhea), it is crucial to acknowledge previously reported adverse effects possibly associated with FMT [16, 17]. A previous study reported two cases of FMT-associated ESBL-producing Escherichia coli bacteremia in the United States, which led the US Food and Drug Administration to mandate additional screening against multidrug-resistant organisms (MDRO) [16]. Therefore, we rigorously screened for viral, bacterial, and protozoal pathogens to prevent potential pathogen transmission via FMT.

The qualification of 62 candidates to serve as potential FMT donors was assessed from August 2017 through June 2020 (Fig. 1). Forty-six (74\%) candidates were excluded based on the pre-screening questionnaire responses. High body mass index (BMI; >25) was the most common reason for donor exclusion (16 individuals), followed by atopy, asthma, and allergy history (15 individuals). The remaining 16 candidates underwent serological/fecal screening, and four (25\%) failed to meet laboratory test criteria. Of the donor candidates excluded at stage 2 , three candidates (75\%) had abnormal blood test results and one carried ESBL-producing Enterobacteriaceae. Overall, 12 candidates ( $19 \%$ of the initial donor candidate pool) qualified as
FMT donors. Our study outcome was consistent with the donor qualification rates in earlier reports [6, 7, 9].

All fecal material from the qualified donors was processed into slurries and quarantined for one month to ensure that it passed the monthly re-qualification process. The re-qualification process involved the same clinical assessment and laboratory test stages as the initial screening process. After the re-qualification process, nine candidates (75\%) did not meet the laboratory test criteria as they showed risks for infectious diseases (five with MDRO, four with Helicobacter pylori, one with enteric bacteria, and one with Blastocystis hominis colonization; one candidate had a positive UBT result) (Fig. 1). Finally, only three candidates (5\% of the donor candidate pool) were eligible to donate feces. These results suggest that continuous donor recruitment is necessary to prevent a shortage of donors and FMT materials. Earlier reports demonstrated similar donor-screening outcomes, with several candidate donors failing to meet the re-qualification criteria [6, 9]. A high percentage of donor failure on monthly requalification screening of FMT donors emphasizes that such screening could prevent the potential transmission of infectious materials through FMT by identifying risk factors not detected in the initial screening process.

Regular screening of FMT donors is crucial, as it serves as an ongoing safety control measure. H. pylori carriage was not a concern for donor candidate disqualification during the initial donor screening. However, four of the initially qualified donors, accounting for $33 \%$ of the qualified donors, were excluded owing to $H$. pylori carriage during the re-qualification process. Owing to the high prevalence of potentially asymptomatic $H$. pylori carriers in the donor pool, our center added UBT to the ongoing screening process in May 2019. Although nested PCR for $H$. pylori detection was used due to its high sensitivity and specificity, possible degradation of $H$. pylori DNA may have contributed to a lower rate of positive test results [18]. UBT, the gold standard for $\mathrm{H}$. pylori diagnosis, was added to the existing donor screening protocol as an additional method for identifying asymptomatic $H$. pylori carriers [18-20]. A larger sample size may be required to obtain a better representation of the healthy population in Korea and determine whether high $H$. pylori prevalence could be a generalizable criterion for donor exclusion in Asian fecal microbiota banks.

To the best of our knowledge, this is the first report to outline the donor screening process and outcomes at a fecal microbiota bank in Asia. After establishing donor screening criteria based on pre-existing protocols and consensus reports, the FMT donor qualification rate was $19 \%$. High $\mathrm{BMI}(>25 ; \mathrm{N}=16)$ and abnor- 


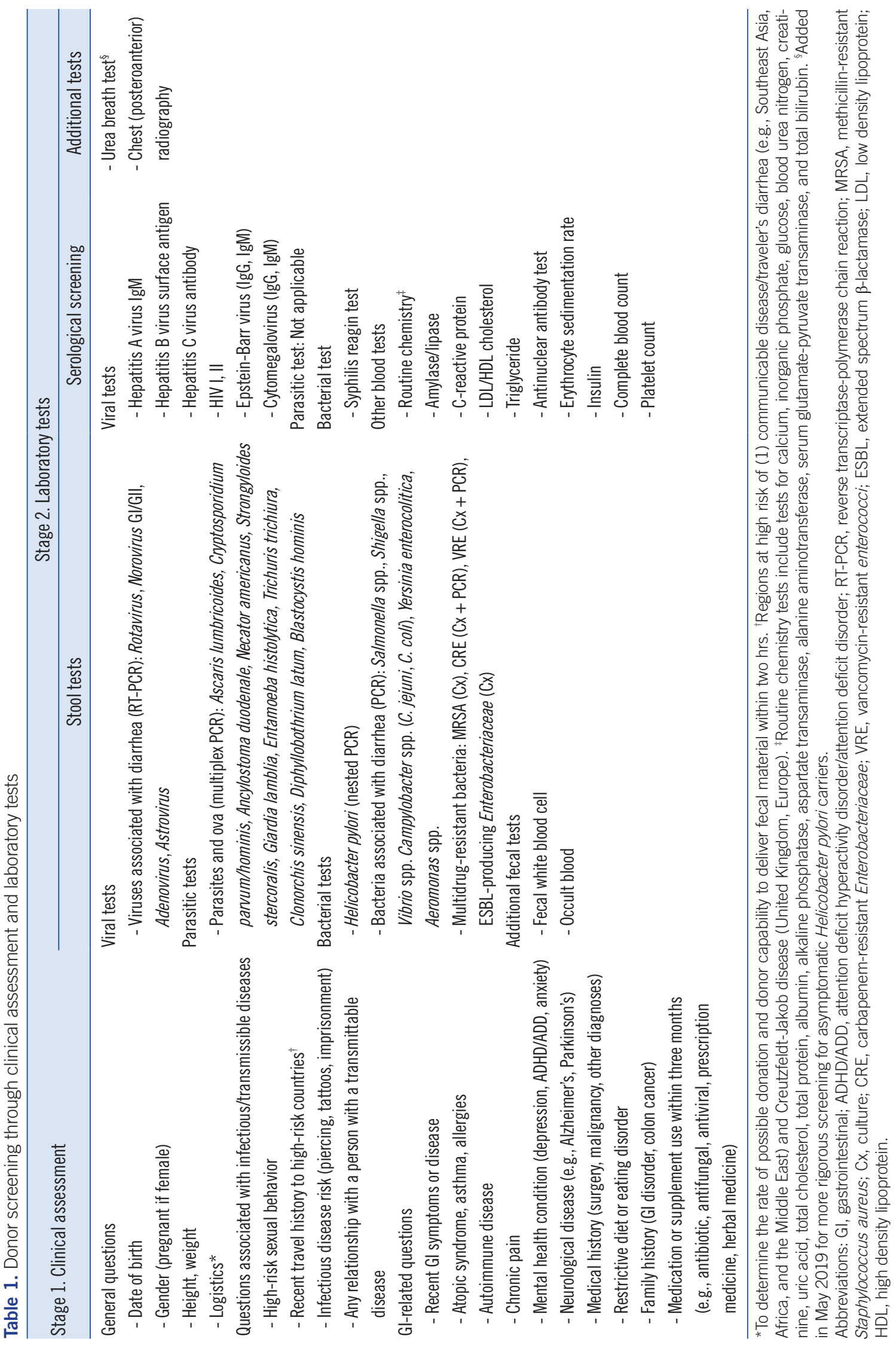




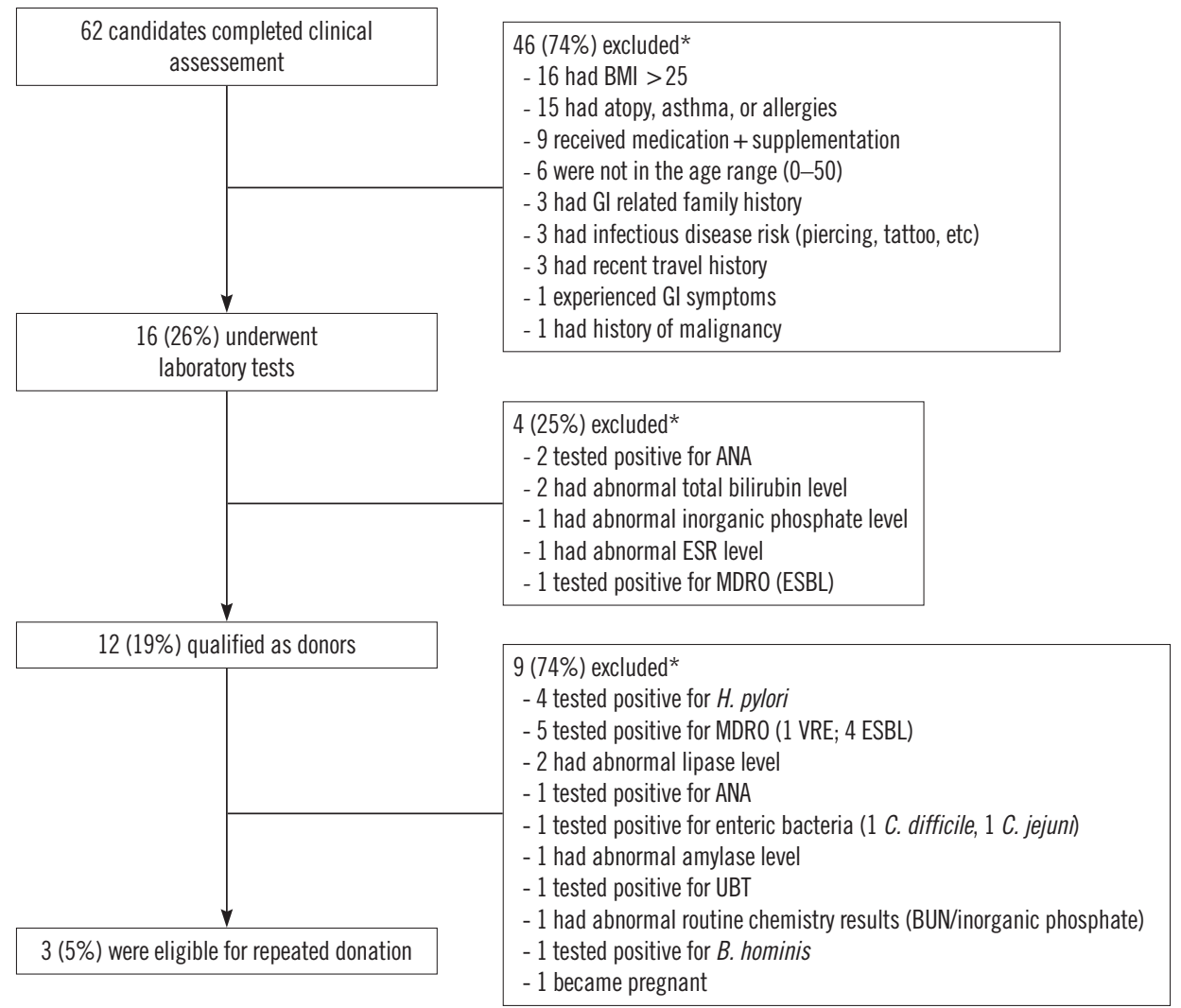

Fig. 1. Fecal microbiota transplantation donor screening process and outcomes. * Some donor candidates met multiple criteria for exclusion. Abbreviations: BMI, body mass index; GI, gastrointestinal; ANA, antinuclear antibody; ESR, erythrocyte sedimentation rate; MDRO, multidrug-resistant organisms; ESBL, extended spectrum $\beta$-lactamase; VRE, vancomycin-resistant enterococci; UBT, urea breath test; BUN, blood urea nitrogen.

mal serological test results (increased total bilirubin level $(2.2$ $\mathrm{mg} / \mathrm{dL}$ ) and positive antinuclear antibody test; $\mathrm{N}=4$ ) were the major criteria for donor exclusion based on clinical assessment and laboratory tests, respectively. A monthly donor re-qualification process contributed to a high overall donor exclusion rate. It resulted in identifying new risk factors, including carriage of infectious agents ( $H$. pylori and B. hominis) and abnormal lipase and amylase levels, that were not considered during the initial donor screening process for FMT.

\section{ACKNOWLEDGEMENTS}

Part of this work was presented at the Laboratory Medicine Congress \& Exhibition 59th annual meeting, November 2018. We especially wish to thank Professors Jae Hee Cheon, Jun Yong Choi, Su Jin Jeong, Hong Ko, Sang Kil Lee, Moo Suk Park, and Soo Jung Park from Yonsei University College of Medicine for collaborative discussions regarding the development of the FMT donor screening protocol as well as the laboratory staff of the Department of Laboratory Medicine at Severance Hospital for assis- tance with the laboratory examination of donor stool samples.

\section{AUTHOR CONTRIBUTIONS}

Chin HS, Kim YH, and Yong D designed the study; Seo HS, Chin HS, Moon HS, Kim YH, and Kim KN collected the clinical samples and analyzed and interpreted the data; and Seo HS, Nguyen LP, and Yong D wrote the manuscript. All authors read and approved the final manuscript.

\section{CONFLICTS OF INTEREST}

Seo HS, Chin HS, Moon HS, Kim KN, and Yong D are partly employed by Microbiotix Corporation. Kim YH is a former employee of Microbiotix Corporation. No other potential conflicts of interest relevant to this article were reported.

\section{RESEARCH FUNDING}

This study was supported by the Korea Health Technology R\&D 
Project through the Korea Health Industry Development Institute (KHIDI), funded by the Ministry of Health \& Welfare, Korea (grant No.: HI14C1324); the Korea Institute of Planning and Evaluation for Technology in Food, Agriculture, Forestry and Fisheries (IPET) through the Agricultural Microbiome R\&D Program, funded by the Ministry of Agriculture, Food and Rural Affairs (MAFRA)(918003-4); and a clinical research grant from Microbiotix Inc.

\section{ORCID}

Hyun Soo Seo

Hyung Sun Chin

Yeon-Hee Kim

Hye Su Moon

Kyungnam Kim

Le Phuong Nguyen

Dongeun Yong https://orcid.org/0000-0003-0658-4724

https://orcid.org/0000-0002-0468-2729

https://orcid.org/0000-0002-8595-2427

https://orcid.org/0000-0002-2857-6306

https://orcid.org/0000-0002-4889-3069

https://orcid.org/0000-0001-6123-5426

https://orcid.org/0000-0002-1225-8477

\section{REFERENCES}

1. Dinh A, Fessi H, Duran C, Batista R, Michelon H, Bouchand F, et al. Clearance of carbapenem-resistant Enterobacteriaceae vs vancomycinresistant enterococci carriage after faecal microbiota transplant: a prospective comparative study. J Hosp Infect 2018;99:481-6.

2. Kao D, Roach B, Silva M, Beck P, Rioux K, Kaplan GG, et al. Effect of oral capsule- vs colonoscopy-delivered fecal microbiota transplantation on recurrent Clostridium difficile infection: a randomized clinical trial. JAMA 2017;318:1985-93.

3. Sokol H, Landman C, Seksik P, Berard L, Montil M, Nion-Larmurier I, et al. Fecal microbiota transplantation to maintain remission in Crohn's disease: a pilot randomized controlled study. Microbiome 2020;8:12.

4. Cammarota G, laniro G, Tilg H, Rajilić-Stojanović M, Kump P, Satokari R, et al. European consensus conference on faecal microbiota transplant in clinical practice. Gut 2017;66:569-80.

5. Costello SP, Tucker EC, La Brooy J, Schoeman MN, Andrews JM. Establishing a fecal microbiota transplant service for the treatment of Clostridium difficile infection. Clin Infect Dis 2016;62:908-14.

6. Craven LJ, Nair Parvathy S, Tat-Ko J, Burton JP, Silverman MS. Extended screening costs associated with selecting donors for fecal microbiota transplantation for treatment of metabolic syndrome-associated diseases. Open Forum Infect Dis 2017;4:ofx243.
7. Kassam Z, Dubois N, Ramakrishna B, Ling K, Qazi T, Smith M, et al. Donor screening for fecal microbiota transplantation. N Engl J Med 2019;381:2070-2.

8. Rode AA, Bytzer P, Pedersen OB, Engberg J. Establishing a donor stool bank for faecal microbiota transplantation: methods and feasibility. Eur J Clin Microbiol Infect Dis 2019;38:1837-47.

9. Terveer EM, van Beurden YH, Goorhuis A, Seegers JFML, Bauer MP, van Nood E, et al. How to: establish and run a stool bank. Clin Microbiol Infect 2017;23:924-30.

10. Woodworth $\mathrm{MH}$, Carpentieri C, Sitchenko KL, Kraft CS. Challenges in fecal donor selection and screening for fecal microbiota transplantation: a review. Gut Microbes 2017;8:225-37.

11. U.S. Food and Drug Administration. Important safety alert regarding use of fecal microbiota for transplantation and risk of serious adverse reactions due to transmission of multi-drug resistant organisms. https:// www.fda.gov/vaccines-blood-biologics/safety-availability-biologics/important-safety-alert-regarding-use-fecal-microbiota-transplantation-andrisk-serious-adverse (Updated on June 2019).

12. Cammarota G, laniro G, Kelly CR, Mullish BH, Allegretti JR, Kassam Z, et al. International consensus conference on stool banking for faecal microbiota transplantation in clinical practice. Gut 2019;68:2111-21.

13. Gweon TG, Lee KJ, Kang DH, Park SS, Kim KH, Seong HJ, et al. A case of toxic megacolon caused by Clostridium difficile infection and treated with fecal microbiota transplantation. Gut Liver 2015;9:247-50.

14. Yoon YK, Suh JW, Kang EJ, Kim JY. Efficacy and safety of fecal microbiota transplantation for decolonization of intestinal multidrug-resistant microorganism carriage: beyond Clostridioides difficile infection. Ann Med 2019;51:379-89.

15. Chung KS and Ko WY. Transfusion guideline. 4th ed. Cheongju: Division of human blood safety surveillance, Korea Centers for Disease Control \& Prevention, 2016; 3-5.

16. DeFilipp Z, Bloom PP, Torres Soto M, Mansour MK, Sater MRA, Huntley $\mathrm{MH}$, et al. Drug-resistant $E$. coli bacteremia transmitted by fecal microbiota transplant. N Engl J Med 2019;381:2043-50.

17. van Beurden $\mathrm{YH}$, de Groot PF, van Nood E, Nieuwdorp M, Keller JJ, Goorhuis A. Complications, effectiveness, and long term follow-up of fecal microbiota transfer by nasoduodenal tube for treatment of recurrent Clostridium difficile infection. United European Gastroenterol J 2017;5: 868-79.

18. Šeligová B, Lukáč L, Bábelová M, Vávrová S, Sulo P. Diagnostic reliability of nested PCR depends on the primer design and threshold abundance of Helicobacter pylori in biopsy, stool, and saliva samples. Helicobacter 2020;25:e12680.

19. Hooi JKY, Lai WY, Ng WK, Suen MMY, Underwood FE, Tanyingoh D, et al. Global prevalence of Helicobacter pylori infection: systematic review and meta-analysis. Gastroenterology 2017;153:420-9.

20. Sung H, Kim MN, Yong D, Lee M, Lee J, Lee MK, et al. Multicenter study on the association of positive Helicobacter pylori stool antigen to anemia in children. Ann Clin Microbiol 2018;21:58-63. 